\title{
Higher Dimensional Strange Quark Matter Coupled to the String Cloud with Electromagnetic Field Admitting One Parameter Group of Conformal Motion
}

\author{
PRADHAN Anirudh 11, KHADEKAR G. S. ${ }^{2}$, MISHRA, Mukesh ${ }^{3}$ and \\ KUMBHARE, Saroj ${ }^{4}$ \\ ${ }^{1,3}$ Department of Mathematics, Hindu Post-graduate College, Zamania-232 \\ 331, Ghazipur, India \\ ${ }^{1}$ E-mail : pradhan@iucaa.ernet.in \\ ${ }^{3}$ E-mail : mukeshkr.mishra@rediffmail.com \\ ${ }^{2,4}$ Department of Mathematics, R. T. M. Nagpur University, Mahatma \\ Jyotiba Phule Educational Campus, Amravati Road, Nagpur-440 033, India \\ ${ }^{2}$ E-mail : gkhadekar@yahoo.com \\ ${ }^{4}$ E-mail : sarojk_11@yahoo.co.in
}

\begin{abstract}
In this paper, we have solved Einstein's field equations in higher dimensional spherically symmetric space-time with strange quark matter attached to the string cloud, assuming one parameter group of conformal motions. The solutions match with the higher dimensional Reissner Nordström metric on the boundary at $r=r_{0}$. The features of the solutions are also discussed in the framework of higher dimensional space-time.
\end{abstract}

Keywords : String, Higher dimension, Starnge quark matter, Conformal motion PACS: 98.80.Cq, 04.20.-q

\section{Introduction}

The exact physical situation at very early stages of the formation of our universe provoked great interest among researcher and it is still a challenging problem for them. It is generally assumed that during the phase transition the symmetry of the universe is broken spontaneously. These strings arise during the phase transition after the big bang explosion as the temperature goes down below some critical temperature as predicted by grand unified theories [1]-6]. The general treatment of strings was initiated by Letelier [7, 8, and Stachel [9]. Of all these cosmological structures, cosmic strings have provoked the most interest. The present day configurations of the universe are not contradicted by

\footnotetext{
${ }^{1}$ Corresponding author
} 
the large scale network of strings in the early universe. Moreover, they may act as gravitational lenses and may give rise to density fluctuations leading to the formations of galaxies [6, 10]. Strings possess stress energy and are coupled to the gravitational field.

Higher dimensional space-time is an active research in its attempt to unify gravity with other forces in nature. This idea is particularly important in the field of cosmology since one knows that our universe was much smaller in its early stage than it is today. In this connection a number of attempts have been made to study the role of gravity with other fundamental forces in nature. The most famous five dimensional theory proposed by Kaluza [11 and Klein 12, was the first theory in which gravitation and electromagnetism could be unified in a single geometrical structure. To achieve unification of all interactions including weak and strong forces, many authors [13-16] have extended the Kaluza-Klein formalism to higher dimensions. In recent years investigations of super-string theory and super gravitational theory have created renewed interest among theoretical physicists to study the physics in higher dimensional spacetime [17, 18. A number of authors (refer [19]- 27] and references therein) have studied the physics of the universe in higher dimensional space-time.

A great deal of attention has recently been paid to strange quark matter. In this study, we will attach strange quark matter to the string cloud. It is plausible to attach strange quark matter to the string cloud. This is because one of such transitions during the phase transitions of the universe could be Quark Gluon Plasma (QGP) $\rightarrow$ hadron gas (called quark-hadron phase transition) when cosmic temperature was $T \sim 200 \mathrm{MeV}$. Recently some authors 28] -31 ] have studied strange quark matter in the different context.

Typically, strange quark matter is modeled with an equation of state (EQS) bases on the phenomenological bag model of quark matter, in which quark confinement is described by an energy term proportional to the volume. In this model, quarks are though as degenerate Fermi gases, which exist only in region of space endowed with a vacuum energy density $B_{c}$ (called as the bag constant). Also, in the framework of this model, the quark matter is composed of massless $\mathrm{u}, \mathrm{d}$ quarks, massive s quarks and electrons. In the simplified version of the bag model, assuming quarks are massless and non-interacting, we then have quark pressure $p_{q}=\rho_{q} / 3$ ( $\rho_{q}$ is the quark energy density); the total energy density is $\rho=\rho_{q}+B_{c}$ but total pressure is $p=p_{q}-B_{c}$.

In this paper we examined charged strange quark matter attached to the string cloud in the higher dimensional spherical symmetric space-time admitting one parameter group of conformal motions. For this purpose, we have solved Einstein's field equations for spherical symmetric space-time with charged strange quark matter attached to the string cloud via conformal motions. This work is the generalization of the work obtained earlier by Yavuz et al. 28. The paper is organized as follows. In section 2, higher dimensional Einstein field equations are obtained for charged strange quark matter attached to the string cloud in spherical symmetric space-time. In section 3, the same Einstein field equations are solved for the same matter via conformal motion depending on conformal vector i.e. $\psi\left(x^{\alpha}\right)$. In section 4 we summarize our results. 


\section{The Metric and Field Equations}

We consider the higher dimensional spherically symmetric line element in the form

$$
d s^{2}=e^{\nu(r)} d t^{2}-e^{\lambda(r)} d r^{2}-r^{2} d \Omega^{2}
$$

with

$$
\begin{gathered}
d \Omega^{2}=d \theta_{1}^{2}+\sin ^{2} \theta_{1} d \theta_{2}^{2}+\sin ^{2} \theta_{1} \sin ^{2} \theta_{2} d \theta_{3}^{2}+\ldots \ldots+\prod_{i=1}^{n-1} \sin ^{2} \theta_{i} d \theta_{n}^{2}, \\
x^{1,2,3, \ldots,(n+1),(n+2)}=r, \theta_{1}, \theta_{2}, \ldots, \theta_{(n+1)}, t .
\end{gathered}
$$

The total energy-momentum tensor $T_{a b}$ is assumed to the sum of the two parts, $T_{a b}^{S}$ and $T_{a b}^{E}$, for string cloud and electromagnetic contributions, respectively, i.e.

$$
T_{a b}=T_{a b}^{S}+T_{a b}^{E}
$$

The energy momentum tensor for string cloud given by Letelier [7]

$$
T_{a b}^{S}=\rho U_{a} U_{b}-\rho_{s} X_{a} X_{b}
$$

where $\rho$ being the rest energy density for the cloud of strings with particles attached to them and $\rho_{s}$ the tension density of the strings. Here

$$
\rho=\rho_{p}+\rho_{s}
$$

where $\rho_{p}$ is the particle energy density.

In this paper we take string quark matter energy density instead of particle energy density in the string cloud. Hence Eq. (44) leads to

$$
\rho=\rho_{q}+\rho_{s}+B_{c}
$$

With the help of Eqs. (3) and (5), we have the energy- momentum tensor for string quark attached to the string cloud

$$
T_{a b}^{S}=\left(\rho_{q}+\rho_{s}+B_{c}\right) U_{a} U_{b}-\rho_{s} X_{a} X_{b}
$$

where $U^{a}$ is the $(n+2)$ velocity $U^{a}=\delta_{(n+2)}^{a} e^{-\nu / 2}, X^{a}$ is the unit space like vector in the radial direction $X^{a}=\delta_{1}^{a} e^{-\lambda / 2}$ which represents the strings directions in the cloud, i.e. the direction of anisotropy. The energy-momentum tensor for electromagnetic field reads

$$
T_{a b}^{E}=-\frac{1}{4 \pi}\left(F_{a}^{c} F_{b c}-\frac{1}{4} g_{a b} F_{\alpha \beta} F^{\alpha \beta}\right),
$$

where $F_{a b}$ is the electromagnetic field tensor in terms of the $(n+2)$-potential $A_{a}$ as

$$
F_{a b}=A_{b ; a}-A_{a ; b}
$$


For the electromagnetic field we consider the gauge

$$
A_{a}(0,0,0,0, \ldots(n+1) 0, \phi(r)) .
$$

Einstein-Maxwell equations can be expressed as

$$
\begin{gathered}
R_{a b}-\frac{1}{2} R g_{a b}=8 \pi T_{a b}, \\
F_{a b ; c}+F_{b c ; a}+F_{c a ; b}=0, \\
F_{; b}^{a b}=-4 \pi J^{a},
\end{gathered}
$$

where $J^{a}$ is the $(n+2)$-current density that becomes $J^{a}=\overline{\rho_{e}} U^{a}, \overline{\rho_{e}}$ being the proper charge density.

In such a background the field Eqs. (6) - (10) for the line-element (10) lead to the following system of equations

$$
\begin{gathered}
8 \pi \rho+E^{2}=-e^{-\lambda}\left[\frac{n(n-1)}{2 r^{2}}-\frac{n \lambda^{\prime}}{2 r}\right]+\frac{n(n-1)}{2 r}, \\
-8 \pi \rho_{s}+E^{2}=-e^{-\lambda}\left[\frac{n \nu^{\prime}}{2 r}+\frac{n(n-1)}{2 r^{2}}\right]+\frac{n(n-1)}{2 r^{2}} \\
E^{2}=\frac{e^{-\lambda}}{2}\left[\nu^{\prime \prime}-\frac{\nu^{\prime} \lambda^{\prime}}{2}+\frac{\nu^{\prime 2}}{2}-\frac{(n-1)\left(\lambda^{\prime}-\nu^{\prime}\right)}{r}+\frac{(n-1)(n-2)}{r^{2}}\right] \\
-\frac{(n-1)(n-2)}{2 r^{2}}, \\
{\left[r^{n} E(r)\right]^{\prime}=4 \pi \rho_{e} r^{n},}
\end{gathered}
$$

where prime $\left({ }^{\prime}\right)$ denotes differentiation with respect to $r$, and $E$ is the usual electric field intensity defined as

$$
\begin{gathered}
F_{(n+2) 1} F^{(n+2) 1}=-E^{2}, \quad E(r)=-e^{-(\nu+\lambda) / 2} \phi^{\prime}(r), \\
\phi^{\prime}(r)=F_{1(n+2)}=-F_{(n+2) 1} .
\end{gathered}
$$

The charge density $\rho_{e}$ defined in equation (14) is related to the proper charge density $\overline{\rho_{e}}$ by

$$
\rho_{e}=\overline{\rho_{e}} e^{\lambda / 2} .
$$

\section{Solutions of the Field Equations}

General relativity provides a rich arena to use symmetries in order to understand the natural relation between geometry and matter furnished by Einstein equations. Symmetries of geometrical/physical relevant quantities of this theory are known as collineations. The most useful collineations is conformal killing vectors which provide a deeper insight into the space-time geometry and facilitate generation of exact solutions to the field equations. Yavuz and Yilmaz 
[29] and Yilmaz et al. [30] who have considered inheriting conformal and special conformal Killing vectors, and also curvature inheritance symmetry in the string cosmology (string cloud and string fluid). Baysal et al. [31 have studied conformal collineation in the string cosmology.

Conformal collineation is defined by

$$
£_{\xi} g_{a b}=2 \psi g_{a b}, \quad \psi=\psi\left(x^{a}\right),
$$

where $£_{\xi}$ signifies the Lie derivative along $\xi^{a}$ and $\psi\left(x^{a}\right)$ is the conformal factor. In particular, $\xi$ is a special conformal Killing vector (SCKV) if $\psi_{; a b}=0$ and $\psi_{, a} \neq 0$. Other sub-cases are homothetic vector (HV) if $\psi_{, a}=0$ and $\psi \neq 0$, and Killing vector $(\mathrm{KV})$ if $\psi=0$. Here semicolon (;) and comma (,) denote the covariant and ordinary derivatives, respectively.

To get the deterministic solution, we assume that the space-time admits a one-parameter group of conformal motions i.e.

$$
£_{\xi} g_{a b}=\xi_{a ; b}+\xi_{b ; a}=2 \psi g_{a b},
$$

where $\psi$ is an arbitrary function of r. From Eqs. (10) and (18) and by virtue of spherical symmetry, we get the following expressions:

$$
\begin{gathered}
\xi^{1} \nu^{\prime}=\psi, \quad \xi^{1}=\frac{\psi r}{2}, \\
\lambda^{\prime} \xi^{\prime}+2 \xi_{, 1}^{1}=\psi, \quad \xi^{n+2}=C_{1}=\text { constant }
\end{gathered}
$$

where a comma (, ) denotes partial derivative with respective r. From Eqs. (19) - (20), we obtain

$$
\begin{gathered}
e^{\nu}=C_{2}^{2} r^{2}, \quad e^{\lambda}=\left(\frac{C_{3}^{2}}{\psi}\right)^{2}, \\
\xi^{a}=\frac{\psi r}{2} \delta_{1}^{a}+C_{1} \delta_{n+1}^{a},
\end{gathered}
$$

where $C_{1}$ and $C_{2}$ are constants of integration. Expressions (21) - (22) contain all the implications derived from the existence of the conformal collineation.

Using Eq. (21) into Eqs. (11) - (13), we have

$$
\begin{gathered}
\rho+E^{2}=\frac{n(n-1)}{2 r^{2}}\left[1-\frac{\psi^{2}}{C_{3}^{2}}\right]-\frac{n \psi \psi^{\prime}}{C_{3}^{2} r}, \\
\rho_{s}+E^{2}=-\frac{n \psi^{2}}{C_{3}^{2} r^{2}}\left[1+\frac{(n-1)}{2}\right]+\frac{n(n-1)}{2 r^{2}}, \\
E^{2}=\frac{n \psi \psi^{\prime}}{C_{3}^{2} r}+\frac{n(n-1) \psi^{2}}{2 C_{3}^{2} r^{2}}-\frac{(n-1)(n-2)}{2 r^{2}} .
\end{gathered}
$$

Here we use geometrized unit so that $8 \pi G=c=1$. From equations (5) and (23) - (25), we get

$$
\rho=-\frac{2 n \psi \psi^{\prime}}{C_{3}^{2} r}-\frac{n(n-1) \psi^{2}}{C_{3}^{2} r^{2}}+\frac{(n-1)^{2}}{r^{2}},
$$




$$
\begin{gathered}
\rho_{s}=-\frac{n \psi \psi^{\prime}}{C_{3}^{2} r}+\frac{(n-1)^{2}}{r^{2}}-\frac{n^{2} \psi^{2}}{C_{3}^{2} r^{2}}, \\
\rho_{p}=\rho_{q}+B_{c}=\rho-\rho_{s}=\frac{n}{r}\left(\frac{\psi^{2}}{C_{3}^{2} r}-\frac{\psi \psi^{\prime}}{C_{3}^{2}}\right) .
\end{gathered}
$$

Using (21), the line-element (1) reduces to

$$
d s^{2}=C_{2}^{2} r^{2} d t^{2}-\frac{C_{3}^{2}}{\psi^{2}} d r^{2}-r^{2} d \Omega^{2} .
$$

If the function $\psi$ and an equation of state for the stresses are specified a priori, the problem will be fully determined. So, we will examine the following physically meaningful three cases depending on $\psi(r)$.

\subsection{Case (i):}

If $\psi=C_{4} r$, then from Eqs. (25) - (28) we get

$$
\begin{gathered}
\rho=\rho_{s}=\frac{(n-1)^{2}}{r^{2}}-n(n+1)\left(\frac{C_{4}}{C_{3}}\right)^{2}, \quad \rho_{p}=0, \\
E^{2}=\frac{n(n+1)}{2}\left(\frac{C_{4}}{C_{3}}\right)^{2}-\frac{(n-1)(n-2)}{2 r^{2}},
\end{gathered}
$$

where $C_{4}$ is an integrating constant.

Let us now consider that the charged sphere extends to radius $r_{0}$. Then the solution of Einstein Maxwell equations for $r>r_{0}$ is given by the higher dimensional Reissner- Nordstöm metric as

$$
\begin{gathered}
d s^{2}=\left[1-\frac{2 m}{r^{(n-1)}}+\frac{2 q^{2}}{n(n-1) r^{2(n-1)}}\right] d t^{2} \\
-\left[1-\frac{2 m}{r^{(n-1)}}+\frac{2 q^{2}}{n(n-1) r^{2(n-1)}}\right]^{-1} d r^{2}-r d \Omega^{2},
\end{gathered}
$$

and the radial electric field in higher dimension is

$$
E=\frac{q(r)}{r^{n}},
$$

where $\mathrm{m}$ and $\mathrm{q}$ are the total mass and charge, respectively.

To match the line-element (29) with the Reisoner-Nordstöm metric (33) across the boundary $r=r_{0}$ we require continuity of gravitational potential $g_{a b}$ at $r=r_{0}$

$$
\left(C_{2} r_{0}\right)^{2}=\left(\frac{\psi}{C_{3}}\right)^{2}=1-\frac{2 m}{r_{0}^{(n-1)}}+\frac{2 q^{2}}{n(n-1) r_{0}^{2(n-1)}},
$$

and also we require the continuity of the electric field, which leads to

$$
E\left(r_{0}\right)=\frac{q}{r_{0}^{n}} .
$$


From Eqs. (31) and (35) we get

$$
\frac{q}{r_{0}^{2 n}}=\frac{n(n+1)}{2}\left(\frac{C_{4}}{C_{3}}\right)^{2}-\frac{(n-1)(n-2)}{2 r_{0}^{2}} .
$$

Putting this expression back into (34) we obtain

$$
\frac{m}{r_{0}^{(n-1)}}=\frac{1}{2}+\frac{1}{(n-1)}\left(\frac{C_{4}}{C_{3}}\right)^{2} r_{0}^{2}-\frac{(n-2)}{2 n} .
$$

Also, from Eqs. (31) and (35) we obtain

$$
m=\frac{(2 n-1)}{n(n+1)} r_{0}^{(n-1)}+\frac{2 q^{2}}{n(n-1)(n+1) r_{0}^{2}} .
$$

\subsection{Case (ii):}

If $\psi=\frac{1}{n} \sqrt{(n-1)^{2} C_{3}^{2}+\frac{n^{2} C_{5}}{r^{2 n}}}$, then from Eqs. (25) - (28) we get

$$
\begin{gathered}
\rho=\rho_{p}=\frac{n(n+1) C_{5}}{r^{2(n+1)} C_{3}^{2}}+\frac{(n-1)^{2}}{n r^{2}}, \quad \rho_{s}=0, \\
\rho_{q}=\frac{n(n+1) C_{5}}{r^{2(n+1)} C_{3}^{2}}+\frac{(n-1)^{2}}{n r^{2}}-B_{c}, \\
E^{2}=\frac{(n-1)^{3}}{2 n r^{2}}-\frac{n(n+1) C_{5}}{2 r^{2(n+1)} C_{3}^{2}}-\frac{(n-1)(n-2)}{2 r^{2}},
\end{gathered}
$$

where $C_{5}$ is constant of integration.

\subsection{Case (iii):}

If $\psi=\sqrt{\frac{(n-2)}{n} C_{3}^{2}+\frac{1}{r^{(n-1)}}}$, then from Eqs. (25) - (28) we get the following expressions:

$$
\begin{gathered}
\rho=\frac{(n-1)}{r^{2}}, \quad \rho_{s}=\frac{1}{r}-\frac{n(n+1)}{2 r^{(n+1)} C_{3}^{2}}, \quad E^{2}=0 . \\
\rho_{p}=\frac{(n-1)}{r^{2}}+\frac{n(n+1)}{2 r^{(n+1)} C_{3}^{2}}-\frac{1}{r^{2}}, \\
\rho_{q}=\frac{(n-1)}{r^{2}}+\frac{n(n+1)}{2 r^{(n+1)} C_{3}^{2}}-\frac{1}{r^{2}}-B_{c},
\end{gathered}
$$




\section{Concluding Remarks}

In this paper, we have studied charged strange quark matter attached to the string cloud in the spherically symmetric space-time admitting one parameter group of conformal motion in the context of higher dimensional space-time. The obtained solutions generalize to higher dimensions the well known results in four dimensional space-time. It is observed that the difference is significant at least in the principle to the analogous situation in four dimensional space-time. The solutions reduce to the four dimensional form when $n=2$.

We have observed that $e^{\nu}$ and $e^{\lambda}$ are positive, continuous and non-singular for $r<r_{0}$. For the case (i) and (ii) we have matched our solutions with higher dimensional Reissner-Nordström metric at $r=r_{0}$. In case (i) we have obtained charged geometric string solutions and charged black string solutions (Eqs. (30) and (38) ). In this case we have also obtained the increase of total mass caused by the charge (see Eq. (38)). Also, if $q=0$ we get total mass for non charged black string i.e. higher dimensional Schwarzschild like string. In case (i) and (ii), we obtain the existence of pressure-less charged strange quark matter and non-charged strange quark matter attached to the string cloud. In case (iii), from Eqs. (42)- (44) it is observed that the strange quark matter decreases the energy of the string in the context of higher dimensional space-time.

\section{Acknowledgements}

Authors (A. P. and G. S. K.) would like to thank IMSc., Chennai, India for providing facility where part of this work was carried out.

\section{References}

[1] Zel'dovich Ya B, Kobzarev I Yu and Okun L B 1975 Zh. Eksp. Teor. Fiz. 673

[2] Zel'dovich Ya B (1975) Sov. Phys.-JETP 401

[3] Kibble T W B (1976) J. Phys. A: Math. Gen. 91387

[4] Kibble T W B Phys. Rep. 67183

[5] Everett A E (1981) Phys. Rev. 24858

[6] Vilenkin A (1981) Phys. Rev. D24 2082

[7] Letelier P S (1979) Phys. Rev. D20 1249

[8] Letelier P S (1983) Phys. Rev. D28 2414

[9] Stachel J (1980) Phys. Rev. D21 2171

[10] Zel'dovich Ya B (1980) Mon. Not. R. Astron. Soc. 192663 
[11] Kaluza T (1921) Sitz. Preuss. Acad. Wiss. Phys. Math. K1 966 (1921)

[12] Klein O (1926) Z. Phys. 37895

[13] Thiry Y (1948) C. R. Acad. Sci.(Paris) 226216

[14] Jordon P (1959) Z. Phys. 157112

[15] Appelquist T, Chodos A and Freund P G O (1987) Modern Kaluza-Klein Theories, Addison-Wesley

[16] Witten, E (1984) Phys. Lett. B144 351

[17] Emel'yanov V M et al. (1986) Phys. Rep 1431

[18] S. Weinberg, Physics In Higher Dimensions, World Scientific, Singapore, (1986).

[19] Myers R C and Perry M J (1986) Ann. Phys. 172304

[20] Marciano W J (1984) Phys. Rev. Letts. 52489

[21] Sahdev D (1984) Phys. Rev. D30 2495

[22] Chatterjee S and Bhui B (1990) Mon. Not. R. Astr. Soc. 24757

[23] Chatterjee S and Bhui B (1993) Int. J. Thor. Phys. 32671

[24] Tan Z Q and Shen, Y G (1998) IL Nuovo Cimento 113339

[25] Khadekar G S, Pradhan A and Molaei M R (2006) Int. J. Mod. Phys. D15 95

[26] Khadekar, G S, Pradhan A and Srivastava, D (2006) Astrophys. Space Sci. 305415

[27] Khadekar, G S, Kamdi V, Pradhan A and Otarod S (2007) Astrophys. Space Sci. To appear.

[28] Yavuz I, Yilmaz I and Baysal, H (2005) Int. J. Mod. Phys. D14 1365

[29] Yavuz I and Yilmaz I (1997) Gen. Rel. Grav. 291295

[30] Yilmaz I, Tharhar I, Yavuz I, Baysal, H and Camci U (1999) Int. J. Mod Phys. D8 659

[31] Baysal H, Camci U, Yilmaz I., Tarhan I and Yavuz I (2002) Int. J. Mod Phys. D11 463 